TITLE:

\title{
Chiral and deconfinement transitions in strong coupling lattice QCD with finite coupling and Polyakov loop effects
}

\section{$\operatorname{AUTHOR(S):~}$}

Nakano, Takashi; Miura, Kohtaroh; Ohnishi, Akira

\section{CITATION:}

Nakano, Takashi ... [et al]. Chiral and deconfinement transitions in strong coupling lattice QCD with finite coupling and Polyakov loop effects. Physical Review D 2011, 83(1): 016014.

\section{ISSUE DATE:}

2011-01

URL:

http://hdl.handle.net/2433/138099

RIGHT:

(c) 2011 American Physical Society. 
PHYSICAL REVIEW D 83, 016014 (2011)

\title{
Chiral and deconfinement transitions in strong coupling lattice QCD with finite coupling and Polyakov loop effects
}

\author{
Takashi Z. Nakano, ${ }^{1,2, *}$ Kohtaroh Miura, ${ }^{3}$ and Akira Ohnishi ${ }^{2}$ \\ ${ }^{1}$ Department of Physics, Faculty of Science, Kyoto University, Kyoto 606-8502, Japan \\ ${ }^{2}$ Yukawa Institute for Theoretical Physics, Kyoto University, Kyoto 606-8502, Japan \\ ${ }^{3}$ INFN-Laboratori Nazionali di Frascati, I-00044, Frascati (RM), Italy
}

(Received 11 November 2010; published 27 January 2011)

\begin{abstract}
We investigate chiral and deconfinement transitions in the framework of the strong coupling lattice QCD for color SU(3) with one species of unrooted staggered fermion at finite temperature and quark chemical potential. We take account of the leading order Polyakov loop terms as well as the next-to-nextto-leading order $\left(1 / g^{4}\right)$ fermionic terms of the strong coupling expansion in the effective action. We investigate the Polyakov loop effects by comparing two approximation schemes, a Haar measure method (no fluctuation from the mean field) and a Weiss mean-field method (with fluctuations). The effective potential is obtained in both cases, and we analytically clarify the Polyakov loop contributions to the effective potential. The Polyakov loop is found to suppress the chiral condensate and to reduce the chiral transition temperature at $\mu=0$, and the chiral transition temperature roughly reproduces the Monte Carlo results at $\beta=2 N_{c} / g^{2} \lesssim 4$. The deconfinement transition is found to be the crossover and first order for light $\left(a m_{0} \lesssim 4\right.$ at $\left.\beta=4\right)$ and heavy quark masses, respectively.
\end{abstract}

DOI: 10.1103/PhysRevD.83.016014

\section{INTRODUCTION}

The phase transition in quantum chromodynamics (QCD) at finite temperature $(T)$ and/or quark chemical potential $(\mu)$ is attracting much attention in recent years. When thermodynamic parameters such as $T$ reach to a typical QCD scale, abundantly formed hadrons start to overlap with each other and matter with color charges would appear, thus a quarkgluon plasma (QGP) [1] is expected to emerge at such high $T$ and/or $\mu$. The first principle studies based on the lattice QCD Monte-Carlo (MC) simulations actually indicate a phase transition from hadron phase to QGP around $T \simeq$ 160-190 (MeV) [2]. Various experimental observations and theoretical arguments imply the formation of a strongly coupled QGP in heavy-ion collisions at the relativistic heavy ion collider. This extreme form of matter will be extensively investigated in LHC-ALICE experiments at CERN, where we can simulate the matter evolution in the early universe. We also expect the transition to quark matter when the baryon chemical potential $N_{c} \mu$ overcomes the nucleon mass energy $N_{c} \mu>M_{N}$. The color superconductor could appear at high density due to the attraction between quarks in the color antisymmetric channel [3], and confined dense quark (quarkyonic) matter is also expected from large $N_{c}$ arguments [4]. The forthcoming experiments at FAIR, J-PARC, and the low energy programs at the relativistic heavy ion collider would be informative for these intermediate and high density states of matter, which may be realized in the neutron-star core.

The QCD phase transition has two aspects, the chiral and deconfinement transitions. These transitions are associated

\footnotetext{
*tnakano@yukawa.kyoto-u.ac.jp
}

PACS numbers: $12.38 . \mathrm{Gc}, 11.10 . \mathrm{Wx}, 11.15 . \mathrm{Me}, 25.75 . \mathrm{Nq}$

with the spontaneous breaking of the global symmetry $S U\left(N_{f}\right)_{L} \times S U\left(N_{f}\right)_{R}$ in the chiral limit and the global $Z_{N_{c}}$ symmetry in the heavy quark-mass limit, respectively, where the order parameters are the chiral condensate and the Polyakov loop. Theoretical frameworks describing the dynamics of these order parameters would be mandatory to understand the QCD phase transition and phase diagram. The lattice QCD MC simulation is the most reliable and rigorous framework to investigate the nonperturbative aspects of QCD [5]. Recent MC simulations demonstrate that the two transitions occur simultaneously within the error bars at small or zero chemical potential [6]. There is no clear explanation for the mechanism of coincidence of transitions, and some models suggest that the chiral and deconfinement transitions can start to separate as quark chemical potential becomes large. Unfortunately, MC simulations have the notorious sign problem at finite $\mu$ [7], and cannot answer whether two transitions can be separated or not. As a result, we need to invoke some approximations in QCD or effective models at finite chemical potentials, such as the strong coupling lattice QCD [8-29], the Nambu-Jona-Lasinio model (NJL) [30] and Polyakov loop extended NJL model (PNJL) [31,32], and the chiral random matrix model [33].

Strong coupling lattice QCD (SC-LQCD) can provide a simple and lattice-based description for the chiral and deconfinement transitions at finite $T$ and $\mu$. This method is based on the strong coupling $\left(1 / g^{2}\right)$ expansion, where the link variables are integrated out analytically and it is possible to elucidate important aspects of the QCD phase transitions beyond a contamination of the sign problem. In the pure Yang-Mills theory, the color confinement in vacuum was shown in the strong coupling limit via the area 
law for the Wilson loop [34]. SC-LQCD of the pure YangMills theory with higher order corrections [9] is recently extended to the deconfinement transition study at finite $T$ [10]. At finite $T$, the deconfinement transition was qualitatively explained on the base of the mean-field treatment of the Polyakov loop in the leading order of the strong coupling expansion [35], and higher order corrections on the Polyakov loop action has been investigated recently [10]. The deconfinement transition is also investigated by taking account of the Haar measure contributions to the effective potential [11]. The behavior of the Polyakov loop is investigated also in the weak-coupling regime [36].

We find some pioneering work on the chiral symmetry breaking in vacuum [28] and its restoration at finite $T$ and/ or $\mu$ [12-14], where one can find a sophisticated expression of the effective potential describing the chiral transition in the strong coupling limit [12-14]. It is remarkable that a promising phase diagram structure has been obtained even in the strong coupling limit (SCL) analytically [15-18] and in MC simulation [19]. In our previous work $[20,21]$, we have developed a formulation to evaluate the next-to-leading order [NLO, $\left.\mathcal{O}\left(1 / g^{2}\right)\right][13,20,22]$, and the next-to-next-to-leading order [NNLO, $\left.\mathcal{O}\left(1 / g^{4}\right)\right][21,23]$ of the strong coupling expansion. Finite coupling effects appear as modifications of the quark mass, quark chemical potential, and the wave function renormalization factor. The phase diagram evolution with $\beta=2 N_{c} / g^{2}$ can be interpreted in terms of those modifications. The SCLQCD technique developed in these works is also applied to the graphene system in the condensed-matter physics [37], where the strong coupling gauge theory is applicable at low energies.
In the works mentioned above, either of the two order parameters is included, and the interplay between these order parameters is not discussed. One of the interesting developments can be found in the works by Gocksch and Ogilvie [24] and by Ilgenfritz and Kripfganz [25]. They developed a model including both of the chiral condensate $(\sigma)$ and Polyakov loop $(\ell)$. In this model, abbreviated as the Gocksch-Ogilvie-Ilgenfritz-Kripfganz (GOIK) model, the deconfinement is governed by the effective action for $\ell$ derived in the leading order of the strong coupling expansion in the Pure Yang-Mills theory, and the chiral transition is described by the strong coupling limit effective action for quarks [24-26]. The coupling between $\sigma$ and $\ell$ is found to appear naturally from the quark determinant. This coupling leads to the correlation of the chiral condensate and Polyakov loop, and the two transitions take place at similar temperatures [26]. This understanding in the GOIK model led to the development of the Polyakov loop extended PNJL model [31].

From a view point of the rigorous strong coupling expansion, there is a problem to define the expansion order when we include both the chiral condensate and Polyakov loop; the quark effective action has the strong coupling limit $\mathcal{O}\left(1 / g^{0}\right)$, while the leading order Polyakov loop action is proportional to $1 / g^{2 N_{\tau}}$, where $N_{\tau}$ is the temporal lattice size. We therefore should regard the order of the strong coupling expansion separately in the quark and Polyakov loop effective action. Namely, we start from the leading order in each of the quark and Polyakov loop action, $\mathcal{O}\left(1 / g^{0}\right)$ and $\mathcal{O}\left(1 / g^{2 N_{\tau}}\right)$, respectively, and extend the framework by including higher order terms of the strong coupling expansion in two directions. As shown in Table I,

TABLE I. Classification of SC-LQCD at finite $T$ and $\mu$. The unrooted staggered fermion is utilized in the quark effective action except for [10], where the Wilson fermion is included in the hopping parameter expansion. In Ref. [35], they treat the chiral spin model, which is essentially the same as that of the Polyakov loop action. SCL-LQCD denotes the strong coupling limit of lattice QCD.

\begin{tabular}{|c|c|c|c|}
\hline Chiral quark action & Without Polyakov loop & $\begin{array}{l}\text { Polyakov loop action } \\
\text { Leading order }\end{array}$ & Higher orders \\
\hline w/o quarks & - & $\begin{array}{l}\text { Kogut, Snow, Stone [35] } \\
\text { Polonyi, K. Szlachanyi; } \\
\text { Gross; Bartholomew et al. } \text { [11] }\end{array}$ & Langelage, Münster, Philipsen [10] \\
\hline $\mathrm{LO}\left[\mathrm{SCL}, \mathcal{O}\left(1 / g^{0}\right)\right]$ & “SCL-LQCD” Refs. [12-18,28] & $\begin{array}{c}\text { "GOIK model" } \\
\text { Gocksch, Ogilvie [24] } \\
\text { Ilgenfritz, Kripfganz [25] } \\
\text { Fukushima [26] }\end{array}$ & - \\
\hline $\operatorname{NLO}\left[\mathcal{O}\left(1 / g^{2}\right)\right]$ & $\begin{array}{c}\text { “NLO SC-LQCD” } \\
\text { Faldt, Petersson [13] } \\
\text { Bilic et al. } \text { [22] } \\
\text { Miura, Nakano, Ohnishi, } \\
\text { Kawamoto [20] }\end{array}$ & $\begin{array}{c}\text { “P-SC-LQCD” } \\
\text { Nakano, Miura, Ohnishi } \\
\text { (present work) Miura, } \\
\text { Nakano, Ohnishi [27] }\end{array}$ & - \\
\hline NNLO $\left[\mathcal{O}\left(1 / g^{4}\right)\right]$ & $\begin{array}{c}\text { "NNLO SC-LQCD" } \\
\text { Jolicoeur et al. [23] } \\
\text { Nakano, Miura, Ohnishi [21] }\end{array}$ & & - \\
\hline
\end{tabular}


the effective action adopted in the GOIK model is the starting point, where both of the quark and Polyakov loop effective action terms are in the leading order. In this effective action, the QCD coupling constant appears only in the coefficient of the Polyakov loop action, and that coefficient $J=\left(1 / g^{2} N_{c}\right)^{N_{\tau}}$ is replaced with an expression $J \simeq \exp \left(-\kappa a^{2} / T\right)$ using the string tension $\kappa$ [24-26]. This is not fully consistent with the quark effective action where the coupling constant is assumed to be infinite. Therefore, it would be more favorable to combine the quark effective action in SC-LQCD with finite coupling effects [13,20-23] with the Polyakov loop action. Since the NLO and NNLO effects in SC-LQCD are represented as modifications in the form of the SCL effective action [20,21], we can combine them with the Polyakov loop effects in a similar way to that in the GOIK model. This Polyakov loop extended strong coupling lattice $Q C D(P-S C-L Q C D)$ would be a promising framework to investigate the relation between the chiral and deconfinement transitions, and has some advantages over other effective models. For example, P-SC-LQCD is directly based on the lattice QCD, and we can compare its results with those in $\mathrm{MC}$ simulations at zero chemical potential.

In this paper, we develop a P-SC-LQCD framework by combining the leading order Polyakov loop effective action and NNLO quark effective action. We derive an analytic expression of the effective potential in P-SC-LQCD at finite $T$ and $\mu$, and investigate the chiral and deconfinement phase transitions at $\mu=0$. The partial results of the whole phase diagram structure including finite $\mu$ are reported in Ref. [27]. Here we adopt one species of unrooted staggered fermion which corresponds to four flavors in the continuum limit. The unrooted staggered fermion is suitable to developing an analytic formulation, since its simple structure enables us to obtain the effective potential analytically. The effective action is composed of the fermionic and pure gluonic parts. We adopt the fermionic effective action with finite coupling effects [13,20-23]. The leading order Polyakov loop action is obtained from the $N_{\tau}$ plaquette configuration as shown in Fig. 1. We evaluate the Polyakov loop effects and derive the effective potential in two kinds of methods. One is the Haar measure method where we replace the Polyakov loop with its mean-field value. Instead of integrating out the temporal link variables, the logarithm of the Haar measure is included in the

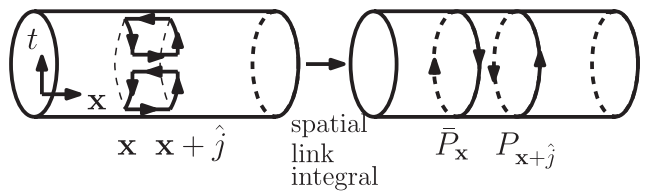

FIG. 1. The leading order of the Polyakov loop effects in the strong coupling expansion. The squares in the left and loops in the right represent the temporal plaquettes and the Polyakov loops, respectively. effective potential. The other is the Weiss mean-field method, which includes the fluctuation effects of the Polyakov loop. In this method, we bosonize the Polyakov loop action and carry out the temporal link integral. On the basis of these effective potentials, we investigate the chiral and deconfinement phase transitions simultaneously. We also compare the results of the two methods.

This paper is organized as follows. In Sec. II, we derive the effective potential with Polyakov loop and finite coupling effects in both Haar measure and Weiss mean-field methods. In Sec. III, we investigate the chiral and deconfinement phase transitions, and compare the results in the two methods. In Sec. IV, we summarize our work and give a future perspective.

Throughout this paper, we utilize the lattice unit $a=1$, and physical values are represented as dimensionless values normalized by the lattice spacing $a$.

\section{EFFECTIVE POTENTIAL}

\section{A. Lattice QCD action}

In the lattice QCD, the partition function and action with one species of unrooted staggered fermion for color $S U\left(N_{c}\right)$ in the Euclidean spacetime are given as

$$
\begin{gathered}
Z_{\mathrm{LQCD}}=\int \mathcal{D}\left[\chi, \bar{\chi}, U_{\nu}\right] e^{-S_{\mathrm{LQCD}},} \\
S_{\mathrm{LQCD}}=S_{F}+S_{G}, \\
S_{F}=\frac{1}{2} \sum_{x} \sum_{\rho=0}^{d}\left[\eta_{\rho, x} \bar{\chi}_{x} U_{\rho, x} \chi_{x+\hat{\rho}}-\eta_{\rho, x}^{-1} \bar{\chi}_{x+\hat{\rho}} U_{\rho, x}^{\dagger} \chi_{x}\right] \\
+m_{0} \sum_{x} \bar{\chi}_{x} \chi_{x}, \\
S_{G}=\frac{2 N_{c}}{g^{2}} \sum_{P}\left[1-\frac{1}{2 N_{c}}\left(U_{P}+U_{P}^{\dagger}\right)\right]
\end{gathered}
$$

where $\chi(\bar{\chi}), m_{0}, U_{\rho, x}$, and $U_{P}$ denote the quark (antiquark) field, the bare quark mass, link variable, and plaquette, respectively. In the staggered fermion, the spinor index does not appear explicitly and its structure is expressed in the staggered phase factor $\eta_{\rho, x}=\left(\eta_{0, x}, \eta_{j, x}\right)=$ $\left(e^{\mu},(-1)^{x_{0}+\cdots+x_{j-1}}\right)[28,38,39]$. The quark chemical potential $\mu$ on the lattice is introduced as a weight of the temporal hopping in the staggered factor [40]. The above lattice QCD action has a global symmetry $U(1)_{V} \times U(1)_{A}$ in the chiral limit $\left(m_{0} \rightarrow 0\right)$, and a staggered chiral transformation is given as $\chi_{x} \rightarrow e^{i \theta \epsilon_{x}} \chi_{x}$ where $\epsilon_{x}=$ $(-1)^{x_{0}+\cdots+x_{d}}$ is related to $\gamma_{5}[28,38,39]$.

Throughout this paper, we consider the case of color $S U\left(N_{c}=3\right)$ in the $3+1$ dimension $(d=3)$ spacetime. Temporal and spatial lattice sizes are denoted as $N_{\tau}$ and $L$, respectively. While $T=1 / N_{\tau}$ takes discrete values, we consider $T$ as a continuous valued temperature. 
We take account of finite $T$ effects by imposing periodic and antiperiodic boundary conditions on link variables and quark fields, respectively. We take the static and diagonalized gauge (called Polyakov gauge) for temporal link variables with respect for the periodicity [12]. In these setups, we derive the effective potential $\mathcal{F}_{\text {eff }}=$ $-\log \left[Z_{\mathrm{LQCD}}\right] /\left(N_{\tau} L^{d}\right)$ on the basis of the strong coupling expansion with Polyakov loop effects.

\section{B. Effective action with Polyakov loop effects}

To treat the chiral and deconfinement phase transitions simultaneously, we evaluate the leading order Polyakov loop effects in the pure Yang-Mills sector and NNLO effects in the fermionic sector. In a finite $T$ treatment of SC-LQCD, we first derive an effective action from the spatial link $\left(U_{j}\right)$ integrals, and the temporal link $\left(U_{0}\right)$ integral is evaluated later to consider the thermal effects of quarks $[13,14]$.

We consider the leading order Polyakov loop effective action $S_{\text {eff }}^{(P)}$ of the strong coupling expansion in the pure Yang-Mills sector, which is obtained from the spatial link integral of the $N_{\tau}$ plaquettes sequentially connected in the temporal direction as shown in Fig. 1.

$$
S_{\mathrm{eff}}^{(P)}=-\left(\frac{1}{g^{2} N_{c}}\right)^{N_{\tau}} N_{c}^{2} \sum_{\mathbf{x}, j>0}\left(\bar{P}_{\mathbf{x}} P_{\mathbf{x}+\hat{j}}+\text { H.c. }\right)
$$

where $P_{\mathbf{x}}=\operatorname{tr}_{c} \prod_{\tau} U_{0}(\tau, \mathbf{x}) / N_{c}$ represents the Polyakov loop. The factor $1 / g^{2} N_{c}$ arises from the spatial link integral. This effective action shows the nearest-neighbor interaction of the Polyakov loop. While we cannot reach the Stefan-Boltzmann limit in the high temperature limit with $S_{\text {eff }}^{(P)}$ [10], it is found to give results qualitatively consistent with the MC simulations on the first-order phase transition properties such as the deconfinement temperature and the discontinuity of the Polyakov loop in the mean-field treatment of SC-LQCD $[15,35]$, then essential dynamics around the deconfinement transition regime may be described.

As for the effective action for quarks, we utilize the NNLO effective action [21] derived from one- and twoplaquette configurations, which correspond to $\mathcal{O}\left(1 / g^{2}\right)$ and $\mathcal{O}\left(1 / g^{4}\right)$, respectively, in addition to the strong coupling limit terms $\left[\mathcal{O}\left(1 / g^{0}\right)\right]$. We consider only the leading order terms of the $1 / d$ expansion, $\mathcal{O}\left(1 / d^{0}\right)$, which corresponds to taking minimum quark number configurations for a given plaquette geometry [29]. We briefly explain the NNLO effective action in Appendix A.

The effective action including both of the quark and Polyakov loop contributions is represented as

$$
S_{\mathrm{eff}}=S_{\mathrm{eff}}^{(F)}+S_{\mathrm{eff}}^{(X)}+S_{\mathrm{eff}}^{(P)} .
$$

$S_{\text {eff }}^{(F)}$ represents the NNLO fermionic effective action,

$$
\begin{gathered}
S_{\text {eff }}^{(F)}=Z_{\chi} \sum_{x y} \bar{\chi}_{x} G_{x y}^{-1}\left(\tilde{m}_{q} ; \tilde{\mu}, T\right) \chi_{y}, \\
G_{x y}^{-1}\left(m_{q} ; \mu, T\right)=\frac{1}{2}\left[e^{\mu} U_{0, x} \delta_{x+\hat{0}, y}-e^{-\mu} U_{0, x}^{\dagger} \delta_{x-\hat{0}, y}\right]+m_{q} \delta_{x y} .
\end{gathered}
$$

Finite coupling effects result in the modifications of the wave function renormalization factor $Z_{\chi}$, quark mass $\tilde{m}_{q}$, and effective chemical potential $\tilde{\mu}$. $S_{\text {eff }}^{(X)}$ represents the auxiliary field part of the effective action described in Appendix A.

From a naive counting of the strong coupling expansion order, the leading order Polyakov loop action composed of the plaquettes is in the higher order $\left[\mathcal{O}\left(1 / g^{2 N_{\tau}}\right)\right]$, compared with the NNLO terms $\left[\mathcal{O}\left(1 / g^{4}\right)\right]$ stem from the quark sector. As discussed in the Introduction, we set the starting point of chiral and deconfinement dynamics as the effective action of $\mathcal{O}\left(1 / g^{0}\right)$ and $\mathcal{O}\left(1 / g^{2 N_{\tau}}\right)$ in the quark and Polyakov loop sectors, respectively. The effective action in Eq. (6) corresponds to an extension in the quark sector from this starting point.

From these action terms, an approximate QCD partition function is obtained, and the effective potential is defined by the logarithm of the partition function as

$$
\begin{aligned}
\mathcal{F}_{\text {eff }} & \equiv-\frac{1}{N_{\tau} L^{d}} \log \left[\int \mathcal{D}\left[\chi, \bar{\chi}, U_{0}\right] e^{-S_{\text {eff }}^{(F)}-S_{\text {eff }}^{(X)}-S_{\text {eff }}^{(P)}}\right] \\
& =\mathcal{F}_{q}(\Phi ; \mu, T)+U_{g}(\ell, \bar{\ell})+\mathcal{F}_{\text {eff }}^{(X)}(\Phi) .
\end{aligned}
$$

Here $\mathcal{F}_{\mathrm{q}}(\Phi ; \mu, T), U_{g}(\ell, \bar{\ell})$, and $\mathcal{F}_{\text {eff }}^{(X)}(\Phi)$ represent the quark free energy, pure gluonic potential, and the effective potential including only the auxiliary fields, respectively. We obtain $\mathcal{F}_{\mathrm{q}}(\Phi ; \mu, T)$ and $U_{g}(\ell, \bar{\ell})$ by evaluating the Grassmann $(\chi, \bar{\chi})$ integral and the temporal link $\left(U_{0}\right)$ integral,

$$
\begin{aligned}
& \int \mathcal{D} U_{0} e^{-S_{\text {eff }}^{(P)}} \operatorname{Det}\left[Z_{\chi} G^{-1}\left(\tilde{m}_{q} ; \tilde{\mu}, T\right)\right] \\
& =\prod_{\mathbf{x}} e^{N_{c}\left(\log Z_{\chi}+E_{q}\right) / T} \int d \mathcal{U}_{0} e^{-S_{\text {eff }}^{(P)}} \operatorname{det}_{c}\left[\left(1+\mathcal{U}_{0} e^{-\left(E_{q}-\tilde{\mu}\right) / T}\right)\right. \\
& \left.\times\left(1+\mathcal{U}_{0}^{\dagger} e^{-\left(E_{q}+\tilde{\mu}\right) / T}\right)\right],
\end{aligned}
$$

where $\mathcal{U}_{0}(\mathbf{x})=\prod_{\tau} U_{0}(\mathbf{x}, \tau)$. Note that the determinant is for the spacetime and color in the first line of Eq. (10), and for the color in the second line of Eq. (10). In Eq. (10), we carry out these integral as follows $[12,13,17]$. First, we perform the Fourier transformation in the temporal direction and obtain the product in the frequency using the Grassmann integral over the quark fields. Second, we evaluate the product in the frequency by the Matsubara method. Finally, in this paper, we evaluate the temporal link integral in two kinds of schemes, the Haar measure and Weiss mean-field methods. We explain these methods in the next two subsections. 


\section{Haar measure method}

We shall now derive the effective potential with Polyakov loop effects in the Haar measure method ( $\mathrm{H}$ method). In the $\mathrm{H}$ method, we replace the Polyakov loop with a mean-field value and the Haar measure is taken into account in the Polyakov loop potential instead of carrying out the temporal link integral. The contribution to the effective action is

$$
S_{\mathrm{eff}}^{(P)} \simeq-2 \beta_{p} L^{d} \bar{\ell} \ell,
$$

where $\beta_{p}=\left(1 / g^{2} N_{c}\right)^{N_{\tau}} N_{c}^{2} d, \ell=\left\langle P_{\mathbf{x}}\right\rangle$, and $\bar{\ell}=\left\langle\bar{P}_{\mathbf{x}}\right\rangle$. We assume the mean fields $\ell$ and $\bar{\ell}$ are constant and isotropic.

The temporal link integral is represented as the Haar measure in the Polyakov gauge,

$$
\int d \mathcal{U}_{0}=\int d \ell d \bar{\ell} \cdot 27\left[1-6 \ell \bar{\ell}+4\left(\ell^{3}+\bar{\ell}^{3}\right)-3(\ell \bar{\ell})^{2}\right]
$$

The Polyakov gauge is a static and diagonalized gauge for temporal link variables [12], then $\mathcal{U}_{0}$ is given as follows:

$$
\mathcal{U}_{0}(\mathbf{x})=\prod_{\tau} U_{0}(\mathbf{x}, \tau)=\operatorname{diag}_{c}\left(e^{i \theta_{1}}, e^{i \theta_{2}}, e^{i \theta_{3}}\right) .
$$

This Haar measure shows the Jacobian in the transformation from the temporal link variables $\left(U_{0}\right)$ to the Polyakov loop $(\ell, \bar{\ell})$. Therefore, $\mathcal{F}_{\mathrm{q}}(\Phi ; \mu, T)$ and $U_{g}(\ell, \bar{\ell})$ are given as

$$
\begin{gathered}
\mathcal{F}_{q}=-N_{c} E_{q}-T \log R\left(E_{q}-\tilde{\mu}, N_{c} \ell, N_{c} \bar{\ell}\right) \\
\quad-T \log R\left(E_{q}+\tilde{\mu}, N_{c} \bar{\ell}, N_{c} \ell\right)-N_{c} \log Z_{\chi}, \\
R(x, L, \bar{L}) \equiv 1+L e^{-x / T}+\bar{L} e^{-2 x / T}+e^{-3 x / T}, \\
U_{g}=-2 T \beta_{p} \bar{\ell} \ell-T \log \left[1-6 \ell \bar{\ell}+4\left(\ell^{3}+\bar{\ell}^{3}\right)-3(\ell \bar{\ell})^{2}\right],
\end{gathered}
$$

where $\beta_{p}=\left(1 / g^{2} N_{c}\right)^{1 / T} N_{c}^{2} d$. Here we have replaced the $N_{\tau}$ with $1 / T$. Note that we have omitted irrelevant constants. In Eq. (14), the first line represents the vacuum and quark free energy and the second line represents the antiquark free energy and the contribution of the wave function renormalization factor. The quark free energy includes one- and two-quark excitations $\left(e^{-(E-\tilde{\mu}) / T}, e^{-2(E-\tilde{\mu}) / T}\right)$. In the confined phase $(\ell \sim \bar{\ell} \sim 0)$, the one- and two-quark excitations are suppressed and only the color-singlet state contributions remain. The pure gluonic potential $U_{g}$ does not include the fluctuation of the Polyakov loop since we treat the Polyakov loop as the mean field without the temporal link integral. In the PNJL model, this pure gluonic potential is incorporated to express the properties of the deconfinement phase transition [31].

\section{Weiss mean-field method}

Now we shall evaluate the Polyakov loop effects in the Weiss mean-field method (W method). The W method includes some part of the fluctuation effects of the Polyakov loop. We first bosonize the Polyakov loop action by using the Extended Hubbard-Stratonovich (EHS) transformation [20],

$$
e^{\alpha A B} \approx \exp [-\alpha(\bar{\psi} \psi-A \psi-\bar{\psi} B)],
$$

where $\alpha$ denotes a positive constant, and two auxiliary fields are introduced simultaneously, $(\bar{\psi}, \psi)=(\langle A\rangle,\langle B\rangle))$. EHS transformation is a procedure to bosonize the product of different types of composites [20]. The Polyakov loop action in Eq. (5) is linearized as

$$
\begin{aligned}
S_{\text {eff }}^{(P)} & \approx\left(\frac{1}{g^{2} N_{c}}\right)^{N_{\tau}} N_{c}^{2} \sum_{\mathbf{x}, j>0} 2\left(\bar{\ell} \ell-\bar{P}_{\mathbf{x}} \ell-\bar{\ell} P_{\mathbf{x}}\right) \\
& \simeq 2 \beta_{p} L^{d} \bar{\ell} \ell-2 \beta_{p} \sum_{\mathbf{x}}\left(\bar{P}_{\mathbf{x}} \ell+\bar{\ell} P_{\mathbf{x}}\right),
\end{aligned}
$$

where $\beta_{p}, P_{\mathbf{x}}$, and $\bar{P}_{\mathbf{x}}$ are defined in Sec. II B. $\ell$ and $\bar{\ell}$ represent the auxiliary fields for the Polyakov loop, $(\ell=$ $\left.\left\langle P_{\mathbf{x}}\right\rangle, \bar{\ell}=\left\langle\bar{P}_{\mathbf{x}}\right\rangle\right)$. In the last line of Eq. (18), we assume constant and isotropic values for auxiliary fields $\ell$ and $\bar{\ell}$.

After the Grassmann integral, we carry out the temporal link integral in Eq. (10) explicitly in the Polyakov gauge. Since $S_{\text {eff }}^{(P)}$ includes the Polyakov loop $\left(P_{\mathbf{x}}, \bar{P}_{\mathbf{x}}\right)=\left(\operatorname{tr} \mathcal{U}_{0} / N_{c}, \operatorname{tr} \mathcal{U}_{0}^{\dagger} / N_{c}\right)$, we have to include $S_{\text {eff }}^{(P)}$ in the temporal link integral in the $\mathrm{W}$ method. The relevant part of the temporal link integral in Eq. (10) is given as

$$
\begin{aligned}
Z_{P}= & \int d \mathcal{U}_{0} \operatorname{det}_{c}\left[2 \cosh \left(E_{q} / T\right)+\mathcal{U}_{0} e^{\tilde{\mu} / T}+\mathcal{U}_{0}^{\dagger} e^{-\tilde{\mu} / T}\right] \\
& \times \exp \left[\eta \operatorname{tr} \mathcal{U}_{0}^{\dagger}+\bar{\eta} \operatorname{tr} \mathcal{U}_{0}\right] \\
\equiv & e^{N_{c} E_{q} / T} Z_{P}^{\prime} .
\end{aligned}
$$

We have defined $\eta=2 \beta_{p} \ell / N_{c}$ and $\bar{\eta}=2 \beta_{p} \bar{\ell} / N_{c}$. In Appendix B, we explain the detail of the temporal link integral. Eventually, we derive the effective potential,

$$
\begin{aligned}
\mathcal{F}_{q}= & -N_{c} E_{q}-T \log \left(Z_{P}^{\prime} / L_{0}\right)-N_{c} \log Z_{\chi}, \\
Z_{P}^{\prime} / L_{0}= & R\left(E_{q}-\tilde{\mu}, L_{1}, \bar{L}_{1}\right) R\left(E_{q}+\tilde{\mu}, \bar{L}_{1}, L_{1}\right) \\
& +e^{-2 E_{q} / T}\left[\left(1+e^{-2 E_{q} / T}\right)\right. \\
& +\left(2+L_{2}-L_{1} \bar{L}_{1}\right)\left(1+e^{-2 E_{q} / T}\right) \\
& +\left(2 L_{1}+L_{3}-\bar{L}_{1}^{2}\right) e^{-(E-\tilde{\mu}) / T} \\
& \left.+\left(2 \bar{L}_{1}+\bar{L}_{3}-L_{1}^{2}\right) e^{-(E+\tilde{\mu}) / T}\right] \\
& U_{g}(\ell, \bar{\ell})=2 T \beta_{p} \bar{\ell} \ell-T \log L_{0} .
\end{aligned}
$$

$L_{0}, L_{1}, \bar{L}_{1}, L_{2}, L_{3}, \bar{L}_{3}$ are the functions of $\eta$ and $\bar{\eta}$. These functions include the modified Bessel function $I_{n}(2 \sqrt{\bar{\eta} \eta})$ 
and the ratio of $\ell$ and $\bar{\ell}\left(\phi=\frac{1}{2} \log (\bar{\eta} / \eta)=\frac{1}{2} \log (\bar{\ell} / \ell)\right)$. The explicit expressions of them are shown in Appendix B.

Compared with the effective potential in the $\mathrm{H}$ method where the quark contributions are represented as the vacuum, quark, and antiquark parts, Eq. (14), the effective potential is more complicated. While Eq. (22a) represents quark and antiquark parts (including the one- and twoquark excitations, and temporal baryon hopping) as the $\mathrm{H}$ method, we replace $\ell(\bar{\ell})$ with $P_{\mathbf{x}}\left(\bar{P}_{\mathbf{x}}\right)$ in Fig. 2. The combination of the Bessel function accompany the Boltzmann factors in the $\mathrm{W}$ method. Other terms represent the temporal meson hopping [Eq. (22b)], one- or two-quark and oneor two-antiquark excitations [Eq. (22c)], and one-antiquark (one-quark) and two-quark (two-antiquark) excitations [Eqs. (22d) and (22e)], as shown in Fig. 3. We can understand these physical interpretations by considering the Boltzmann factors. We explain the one-quark and oneantiquark excitations $\left(e^{-2 E_{q} / T}\right)$ in Eq. (22c) as an example. We can decompose Eq. (22a), and find that the contribution of $\left(e^{-2 E_{q} / T}\right)$ comes from the multiplication of one-quark $\left(e^{-\left(E_{q}-\tilde{\mu}\right) / T}\right)$ and one-antiquark $\left(e^{-\left(E_{q}+\tilde{\mu}\right) / T}\right)$ excitations. These contributions of Eqs. (22b)-(22e) in the W method can appear because of the temporal link integral, and not in the $\mathrm{H}$ method or the PNJL model. In the confined phase $(\ell, \bar{\ell} \sim 0)$, the quark excitations are suppressed since $L_{1} \sim$ $\bar{L}_{1} \sim L_{3} \sim \bar{L}_{3} \sim \bar{L}_{2}+2 \sim 0$, and the color-singlet state is dominant. Therefore, Eqs. (22a) and (22b) remain finite in the confined phase, while the other terms, Eqs. (22c)-(22e), vanish.

Note that the pure gluonic potential $U_{g}$ includes the dependence on $\bar{\ell} / \ell$ explicitly (i.e. the dependence on $\mu$ ). When the quark chemical potential is zero $(\mu=0)$, the Polyakov loop for quarks and antiquarks are the same $(\ell=\bar{\ell})$. In comparison, when the quark chemical potential is finite $(\mu \neq 0)$, the Polyakov loop for antiquarks is generally different from that for quarks $(\ell \neq \bar{\ell})$ [41].

In Ref. [35], the pure gluonic potential is derived at $\mu=0$ without quarks. As shown later, we confirmed that our results are consistent with their results, for example, in the temperature of the deconfinement phase transition without quarks.

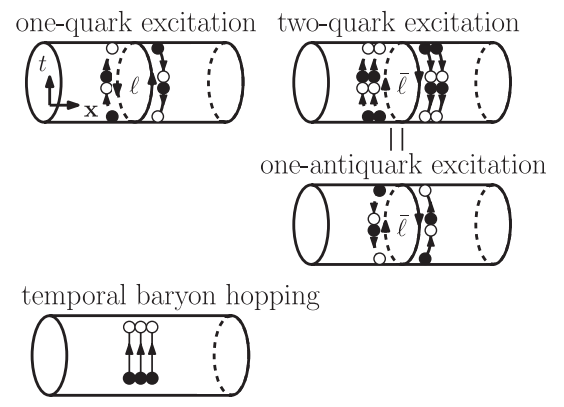

FIG. 2. The quark excitation on the lattice. We show the oneand two-quark excitation in the first line, while the temporal baryon hopping in the third line. (b)

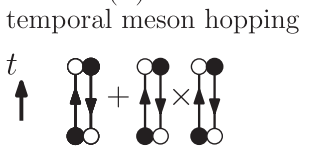

(c)

one-quark one-antiquark

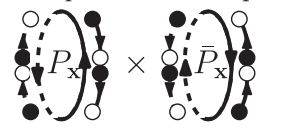

(d)

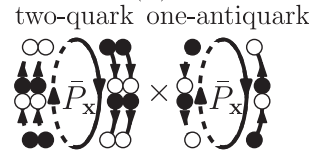

(c)

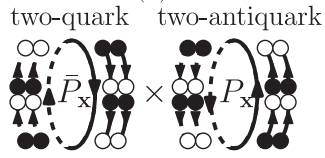

FIG. 3. The panels indicate meson hopping and quark excitation in the $\mathrm{W}$ method: (b) temporal meson hopping; (c) one-quark and one-antiquark excitations, two-quark and two-antiquark excitations; (d) one-antiquark and two-quark excitations. The labels (b), (c), and (d) correspond to Eqs. (22b), (22c), and (22d), respectively.

\section{CHIRAL AND DECONFINEMENT PHASE TRANSITION}

We shall now discuss the phase transitions in P-SCLQCD. We impose the stationary condition for the effective potential with respect to the auxiliary fields, and obtain thermodynamical quantities. In this article, we investigate the chiral and deconfinement transitions at $N_{c}=3$ and $\mu=0$. First, we discuss the deconfinement transition in pure Yang-Mills theory, and next we show the results in the $\mathrm{W}$ method. Finally, we compare the results in the $\mathrm{W}$ and $\mathrm{H}$ methods.

\section{A. Deconfinement transition in pure Yang-Mills theory}

Before discussing the results with quarks, we first examine the deconfinement transition in the pure Yang-Mills theory. In Fig. 4, we compare the Polyakov loop potentials $U_{g}$ in the $\mathrm{W}$ and $\mathrm{H}$ methods, Eqs. (23) and (16), respectively. We show the results at $T=(0.9-1.1) T_{d, c}$ at $\beta=4$ without quark effects. Both of the potentials have qualitatively the same behavior and show the first-order transitions at $T=T_{d, c}$. We find some quantitative differences in these two potentials. First, we note that in the $\mathrm{H}$ method there is a wall at $\ell=1$ where $U_{g}$ diverges. The range of $\ell$ is limited in the $\mathrm{H}$ method to be less than one as can be found from Eq. (16). In the W method, the integral over $\mathcal{U}_{0}$ smoothens the H-method potential,

$$
e^{-U_{g}^{W}(\ell, \bar{\ell}) / T}=\int d \ell^{\prime} d \bar{\ell}^{\prime} e^{-\beta_{P}\left(\ell-\ell^{\prime}\right)\left(\bar{\ell}-\bar{\ell}^{\prime}\right)} e^{-U_{g}^{H}\left(\ell^{\prime}, \bar{\ell}^{\prime}\right) / T}
$$

and the effective potential becomes a milder function of $\ell$. As shown in the dotted line in the upper panel of Fig. $4, U_{g}^{W}$ is smaller (larger) for large (small) $\ell$. Since the transition temperature is more sensitive to the potential at small $\ell$, the $T_{d, c}$ in the $\mathrm{W}$ method is higher than that in the $\mathrm{H}$ method. 
CHIRAL AND DECONFINEMENT TRANSITIONS IN ...

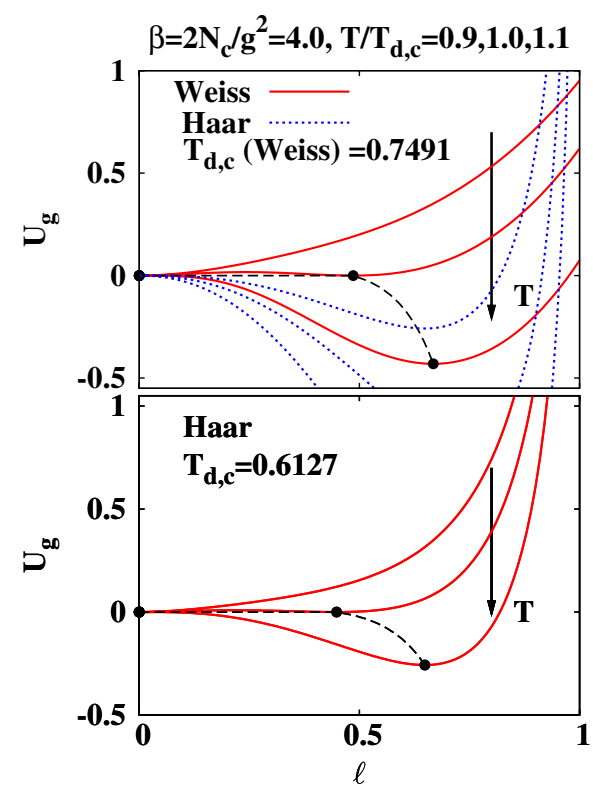

FIG. 4 (color online). The Polyakov loop potential in the W (upper panel) and $\mathrm{H}$ (lower panel) methods. The filled circles show the equilibrium points. We show the results in the lattice unit.

Our treatment in the $\mathrm{W}$ method in the pure Yang-Mills theory is the same as the chiral spin model investigated in Ref. [35]. where the following effective action is adopted:

$$
S_{\mathrm{eff}}^{(K)} / L^{d}=\frac{\beta^{(K)} N_{c}}{4 d} \alpha^{2}-\frac{\beta^{(K)} N_{c}}{2} \alpha(P+\bar{P}) .
$$

When the parameter $\beta^{(K)}$ and the variable $\alpha$ are rewritten according to Table II, this action is found to be equivalent to $S_{\text {eff }}^{(P)}$ in Eq. (18). In Ref. [35], the first-order phase transition is found to take place at the critical coupling $\beta_{c}^{(K)}=0.81$ in the mean-field treatment. By using the relation $\beta^{(K)}=2 \beta_{p} / N_{c} d$, this critical coupling corresponds to $T_{d, c} \sim 0.75$ at $\beta=4$. This value is consistent with our result $T_{d, c} \sim 0.7491$ in the upper panel of Fig. 4.

\section{B. Chiral condensate and Polyakov loop}

We shall now discuss the chiral and deconfinement transitions in the $\mathrm{W}$ method at finite $T$ and $\mu=0$, via the $T$ dependence of the chiral condensate $\sigma=-\langle\bar{\chi} \chi\rangle$ and the Polyakov loop $(\ell, \bar{\ell})=\left(\left\langle P_{\mathbf{x}}\right\rangle,\left\langle\bar{P}_{\mathbf{x}}\right\rangle\right)$. In Fig. 5, we show the quark-mass $\left(m_{0}\right)$ and $T$ dependence of $\sigma$ and $\ell$ at

TABLE II. Correspondence of the parameters between our results and Ref. [35]. Note that we replace $\beta$ in Ref. [35] with $\beta^{(K)}$ to distinguish from $\beta=2 N_{c} / g^{2}$.

\begin{tabular}{lc}
\hline \hline Our results & Kogut et al. [35] $(d=3)$ \\
\hline$\ell$ & $\alpha / 2 d$ \\
$2 \beta_{p} / N_{c}^{2}$ & $2 d \beta^{(K)} /\left(2 N_{c}\right)$ \\
\hline \hline
\end{tabular}

PHYSICAL REVIEW D 83, 016014 (2011)
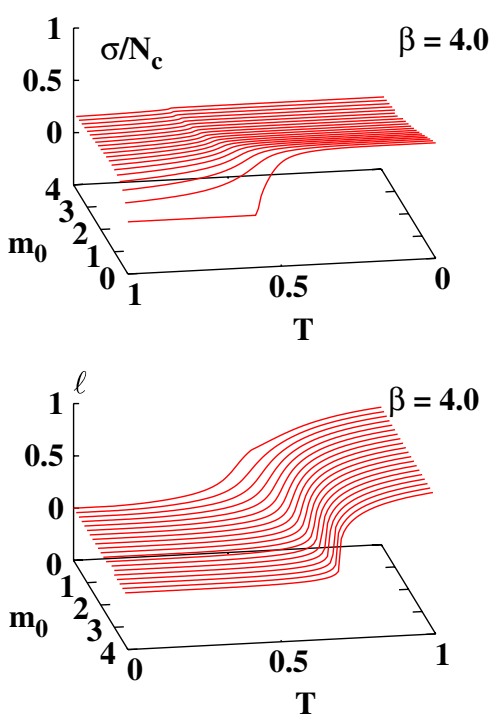

FIG. 5 (color online). The temperature and quark-mass dependence of the chiral condensate (upper panel) and the Polyakov loop (lower panel) in the $\mathrm{W}$ method. We show both of the results in the lattice unit.

$\mu=0$. We adopt the coupling $\beta=4$ as a typical example. As expected, $\sigma$ vanishes sharply at $T_{c}$ at $m_{0}=0$ showing the second order transition in the chiral limit, and $\ell$ starts to show the first-order transition at $m_{0} \sim 4.0$.

In Fig. 6, we show $\sigma$ and $\ell$ as functions of $T$ at $\beta=4$ and $m_{0}=0.05$. Since the quark mass is small but finite, both $\sigma$ and $\ell$ show crossover transition toward the chiral restored $(\sigma \rightarrow 0)$ and deconfined $(\ell \rightarrow 1)$ matter, i.e., a quark-gluon plasma.

From Fig. 6, the chiral and deconfinement transitions seem to take place almost at the same temperatures. In the upper panel of Fig. 7, we show $-d \sigma / d T$ and $d \ell / d T$ as functions of $T$. Both $-d \sigma / d T$ and $d \ell / d T$ are peaked at around $T \sim 0.55$. We here define the chiral $T_{\chi, c}$ and deconfinement $T_{d, c}$ transition temperatures as the peak temperatures of $-d \sigma / d T$ and $d \ell / d T$. In the lower panel of Fig. 7, we display $T_{\chi, c}$ and $T_{d, c}$ as functions of $\beta$.

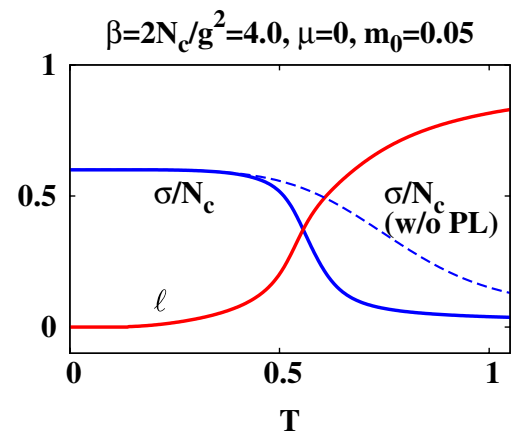

FIG. 6 (color online). The chiral condensate and Polyakov loop in P-SC-LQCD (solid lines), and chiral condensate in SC-LQCD without the Polyakov loop effects (dashed line) as functions of $T$ at $\mu=0$ in the $\mathrm{W}$ method. We show the results in the lattice unit. 
TAKASHI Z. NAKANO, KOHTAROH MIURA, AND AKIRA OHNISHI
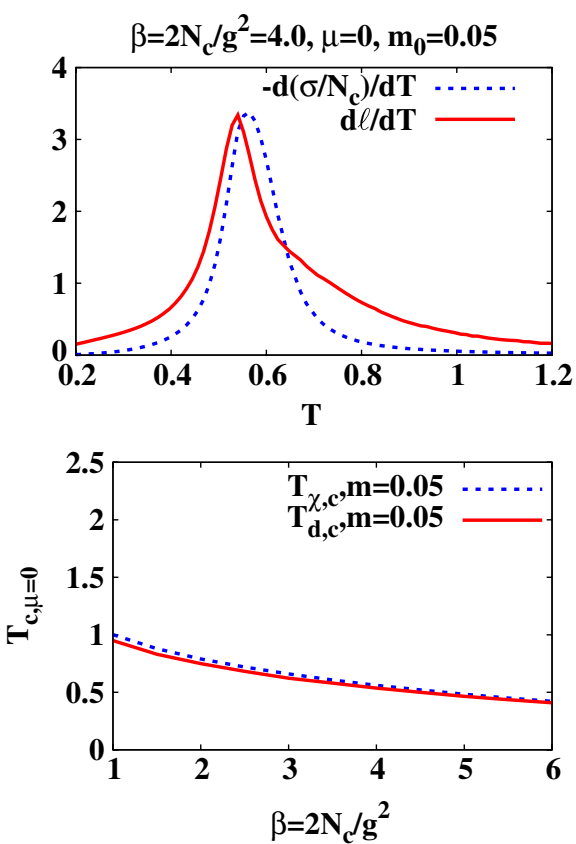

FIG. 7 (color online). Upper panel: The temperature dependence of $-d \sigma / d T$ (dashed line) and $d \ell / d T$ (solid line). Lower panel: $\beta$ dependence of the critical temperatures for the chiral (dashed line) and deconfinement (solid line) phase transitions. All of them are obtained in the $\mathrm{W}$ method. We show both of the results in the lattice unit.

The Polyakov loop is found to suppress the chiral condensate and to reduce the chiral transition temperature in P-SC-LQCD. In Figs. 6 and 8, we compare the chiral condensate $\sigma$ and the chiral transition temperature $T_{\chi, c}$ with and without Polyakov loop effects. We find that both $\sigma$ and $T_{\chi, c}$ in P-SC-LQCD are smaller than those



FIG. 8 (color online). A comparison of P-SC-LQCD (solid line) and SC-LQCD without the Polyakov loop (dashed line) in the lattice unit. We show the results of Weiss mean-filed method (bold solid line) and Haar measure method (thin solid line) in P-SC-LQCD at $m_{0}=0.0$. The triangles represent the results of the critical temperature $\left(T_{c, \mu=0}\right.$, open triangle) and the critical coupling ( $\beta_{c}$, filled triangles) obtained in Monte-Carlo simulations with one species of unrooted staggered fermion: From the left, $T_{c, \mu=0}$ in the SCL with monomer-dimer-polymer simulations [19], $\beta_{c}$ at $\left(N_{\tau}, m_{0}\right)=(2,0.025)$ [44], (2, 0.05) [44], $(4,0.1)[45],(4,0.05)[46,47]$, and $(8,0.0)[48]$. without Polyakov loop effects; the chiral condensate becomes smaller when the Polyakov loop takes a finite value, and the chiral symmetry restoration takes place at lower $T$ by the Polyakov loop effects. In SC-LQCD without Polyakov loop effects, we find the contribution only from color-singlet states as discussed before, then we implicitly assume that the quarks are confined at any $T$. In the $\mathrm{W}$ method, we have one- and two-quark contributions as well when the Polyakov loop takes a finite value. Quark excitation generally breaks the chiral condensates, then it promotes the chiral symmetry to be restored at lower $T$. Similar behavior is found also in the $\mathrm{H}$ method.

This behavior is different from that in the PNJL model $[31,32]$. In PNJL, the Polyakov loop pushes up the chiral transition temperature from that in the NJL model. This difference can be understood as follows. Quarks are deconfined in NJL, then the role of the Polyakov loop is to confine quarks at low $T$ in PNJL. By comparison, only color-singlet hadronic states contribute to the effective potential and quarks are confined in SC-LQCD without $\mathrm{PL}$, then the role of the Polyakov loop is to deconfine quarks at high $T$ in $P$-SC-LQCD. As a result, confined and deconfined phases appear at low and high $T$ region in both PNJL and P-SC-LQCD, and the upward and downward shifts of $T_{c}$ in PNJL and P-SC-LQCD are understood as the directions to enlarge the region which is promoted by the Polyakov loop. As a result, both PNJL and P-SCLQCD give a consistent picture of the chiral and deconfinement transitions, although the confinement properties of quark matter are different in NJL and SC-LQCD without Polyakov loop effects as shown in Table III.

Note that the confinement picture of quark matter in the PNJL model and P-SC-LQCD is not completely the same. In P-SC-LQCD, colors of quarks in a hadron are confined locally when the Polyakov loop is zero. By comparison the interquark distance in a hadron is not necessarily small in PNJL, since the momentum of quarks are specified.

In our previous work on NLO [20] and NNLO [21] SCLQCD, the critical temperature is calculated to be larger than MC results, and NNLO effects on $T_{\chi, c}$ are found to be small. In Figs. 8 and 9, we show $T_{\chi, c}$ in several treatments of P-SC-LQCD in comparison with the MC results. As already discussed, the chiral transition temperature $T_{\chi, c}$ is reduced by the Polyakov loop effects and roughly explains the $\mathrm{MC}$ results, especially in the region $\beta \lesssim 4$. By comparison, the NNLO effects on $T_{\chi, c}$ at $\mu=0$ are small also

TABLE III. Difference between the SC-LQCD with/without the Polyakov loop (PL) effects, the PNJL, and NJL model.

\begin{tabular}{lcr}
\hline \hline & Low $T$ & High $T$ \\
\hline SC-LQCD without PL & \multicolumn{2}{c}{ confined } \\
NJL & \multicolumn{2}{c}{ deconfined } \\
P-SC-LQCD and PNJL & confined & deconfined \\
\hline \hline
\end{tabular}


CHIRAL AND DECONFINEMENT TRANSITIONS IN ...

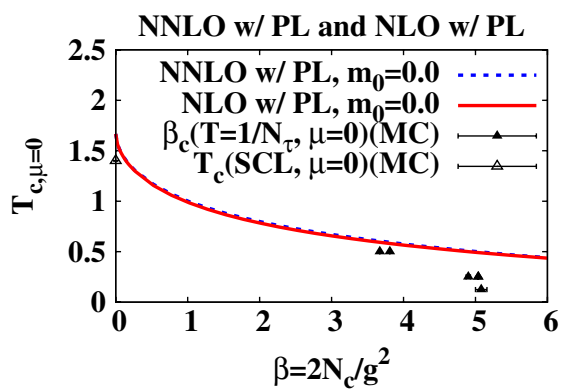

FIG. 9 (color online). A comparison of the chiral transition temperature $T_{\chi, c}$ in P-SC-LQCD with NNLO (solid line) and NLO (dashed line) finite coupling corrections for quarks. The triangles represent the same MC results in Fig. 8. We show the results in the lattice unit.

in the case with Polyakov loop effects. This observation implies that introducing the Polyakov loop, the deconfinement order parameter, is essential to explain the QCD phase transition temperature. In order to explain further reduction of $T_{\chi, c}$ at $\beta \gtrsim 4$, we may need to include higher order terms of $1 / g^{2}$ in the Polyakov loop effective action.

\section{Comparison of Weiss mean-field and Haar measure methods}

The Haar measure method is useful to include the confinement effects in chiral effective models, and it has been widely applied, for example, in the PNJL [31] and PQM [42] models. In this subsection, we discuss the results of the Haar measure method, and compare the results of two methods.

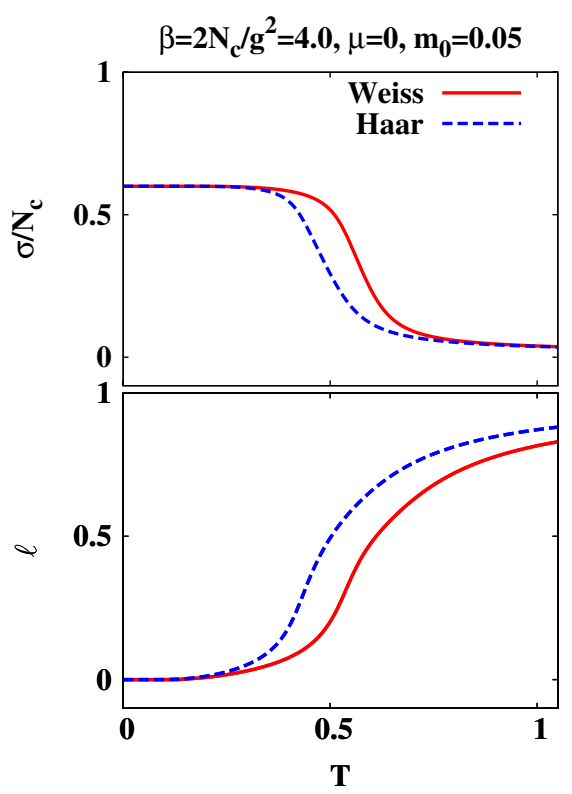

FIG. 10 (color online). The chiral condensate and Polyakov loop as functions of $T$ at $\mu=0$ in the $\mathrm{W}$ and $\mathrm{H}$ methods. The notation is the same as Fig. 6. We show the results in the lattice unit.
PHYSICAL REVIEW D 83, 016014 (2011)

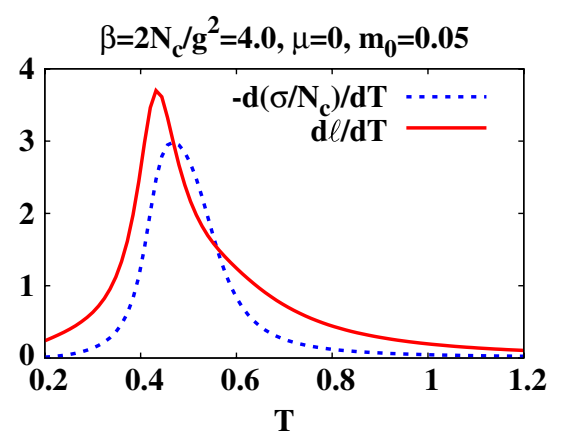

FIG. 11 (color online). Th temperature dependence of $-d \sigma / d T$ (dashed line) and $d \ell / d T$ (solid line) in the Haar measure method ( $\mathrm{H}$ method). The notation is the same as Fig. 7. We show the results in the lattice unit.

First, we compare the chiral condensate and the Polyakov loop in the $\mathrm{W}$ and $\mathrm{H}$ methods. In Fig. 10, we show the $T$ dependence of $\sigma$ and $\ell$ in the two methods. While qualitative behaviors are the same in both of the methods, the chiral condensate (Polyakov loop) in the $\mathrm{W}$ method is larger (smaller) than those in the H method, suggesting that the $\mathrm{W}$ method exhibits a little larger $T_{c}$ than the $\mathrm{H}$ method. In Fig. 8, we compare the chiral transition temperature $T_{\chi, c}$ in the two methods. The transition temperature is defined from the peaks of $-d \sigma / d T$ and $d \ell / d T$ as shown in Fig. 11.

Physically, the temporal link integral in the Weiss meanfield method favors the color-singlet states and therefore


FIG. 12 (color online). Upper panel: The Polyakov-quark coupling $L_{1}$ and $N_{c} \ell$ as functions of $\ell$ at $\mu=0(T=0.5, \ldots, 1.0)$. The dots represent the value of $L_{1}$ under the stationary condition $\left(m_{0}=0.05\right)$. Lower panel: Stationary value of the Polyakovquark coupling $L_{1}$ and $N_{c} \ell$ as functions of the normalized temperature $T / T_{c}$. We show both of the results in the lattice unit. 
suppresses the quark excitation. Also, as another aspect, the smaller Polyakov-quark coupling in the $\mathrm{W}$ method is responsible in part for the larger $T_{c}$. In the $\mathrm{W}$ method, we carry out the temporal link integral of the effective action in which fermions couple with the temporal link variables. This "averaging" procedure as shown Eq. (24) smears the Polyakov-quark coupling. In the upper panel of Fig. 12, we show the comparison of the Polyakov-quark couplings, $L_{1}\left(=\bar{L}_{1}\right)$ and $N_{c} \ell$ in the $\mathrm{W}$ and $\mathrm{H}$ methods, respectively, defined as the factor accompanied with the Boltzmann factor. At $\mu=0$, we find that the stationary value of the coupling is smaller in the W method, $L_{1}\left(=\bar{L}_{1}\right)<N_{c} \ell$, at any temperature as shown in Fig. 12. These observations confirm the smaller Polyakov-quark coupling. Similar behavior is also found in the NJL model with the Polyakov integration [43].

\section{CONCLUDING REMARKS}

In this paper, we have derived an analytic expression of the effective potential at finite temperature and chemical potential in the strong coupling lattice QCD with the Polyakov loop effects using one species of unrooted staggered fermion. We have discussed the chiral and deconfinement transitions at $\mu=0$, especially the Polyakov loop effects on these transitions. We have considered NNLO effects in the strong coupling expansion and the leading order of the $1 / d$ expansion in the quark effective action. We have adopted the leading order Polyakov loop action, $\mathcal{O}\left(1 / g^{2 N_{\tau}}\right)$, and the temporal link $\left(U_{0}\right)$ integral is evaluated in two methods. One is the Haar measure method, where we replace the Polyakov loop with its constant mean-field without the $U_{0}$ integral. The deconfinement dynamics is taken account of via the Haar measure. Another is the Weiss mean-field method, where we bosonize the effective action using the extended Hubbard-Stratonovich transformation and carry out the temporal link integral explicitly. The fluctuation effects appear as the combination of the modified Bessel function and the difference of $\bar{\ell}$ and $\ell$ in the effective potential. We have found that one- and twoquark excitations are allowed in both methods, when the Polyakov loop takes a finite value at high $T$.

We find that the Polyakov loop reduces the chiral transition temperature, and the obtained transition temperatures roughly explain the MC results in the region $\beta \leqq 4$. The chiral and deconfinement transitions are found to take place at similar temperatures, but the two transition temperatures are slightly different when we define them from the susceptibilities. We have compared the results in the $\mathrm{W}$ and $\mathrm{H}$ methods. These methods exhibit qualitatively the same results, but the Polyakov loop effects are found to be weaker in the $\mathrm{W}$ method. The transition temperature is slightly higher and the Polyakov-quark coupling is smaller in the W method. This is because the temporal link integral in the $\mathrm{W}$ method favors the color-singlet states and leads to the suppression of the quark excitation.
There are several points to be studied further. First, we should investigate the QCD phase diagram in finite $T$ and $\mu$ on the basis of the effective potentials derived in this work. Second, it would be a promising direction to extend the present P-SC-LQCD framework by including higher order terms in $1 / g^{2}$ and $1 / d$ in the quark and Polyakov loop effective action. Third, it is interesting to apply the present effective potential to the chiral effective model, for example, to the PNJL model, in order to include the fluctuation of the Polyakov loop. We replace the Polyakov loop potential with that in Eq. (23) and the factor accompanied with the Boltzmann factor $N_{c} \ell\left(N_{c} \bar{\ell}\right)$ with $L_{1}\left(\bar{L}_{1}\right)$ in Eq. (21). These studies will be helpful to shed light on the QCD phase transitions at finite $T$ and $\mu$.

\section{ACKNOWLEDGMENTS}

We would like to thank Hideo Suganuma and Yoshimasa Hidaka for useful discussion and advice. This work was supported in part by Grants-in-Aid for Scientific Research from MEXT and JSPS (No. 22-3314), the Yukawa International Program for Quark-hadron Sciences (YIPQS), and by Grants-in-Aid for the global COE program "The Next Generation of Physics, Spun from Universality and Emergence" from MEXT.

\section{APPENDIX A: NNLO EFFECTIVE ACTION}

We here briefly introduce the NNLO SC-LQCD effective action [21], which we utilize as the quark part of the P-SC-LQCD. The NNLO effective action is derived from one- and two-plaquette configurations, which correspond to $\mathcal{O}\left(1 / g^{2}\right)$ and $\mathcal{O}\left(1 / g^{4}\right)$, respectively, in addition to the strong coupling limit terms $\left[\mathcal{O}\left(1 / g^{0}\right)\right]$. We consider only the leading order terms of the $1 / d$ expansion, $\mathcal{O}\left(1 / d^{0}\right)$. The spatial link integral leaves a sum over spatial directions, and the energy per bond is assumed to be proportional to $1 / d$ in order to keep the action finite at a large spatial dimension in the $1 / d$ expansion [29]. Since the quark field scales as $d^{-1 / 4}$, the leading order $1 / d$ terms correspond to the minimum quark number configurations for a given plaquette geometry. The NNLO effective action is represented as [21]

$$
S_{\mathrm{eff}}^{(\mathrm{NNLO})}=S_{\mathrm{eff}}^{(F)}+S_{\mathrm{eff}}^{(X)},
$$

$S_{\text {eff }}^{(F)}$ represents the fermionic effective action, shown in Eq. (7).

$$
\begin{aligned}
S_{\mathrm{eff}}^{(F)} & =\frac{1}{2} \sum_{x}\left[Z_{-} V_{x}^{+}-Z_{+} V_{x}^{-}\right]+\sum_{x} m_{q}^{\prime} M_{x} \\
& =Z_{\chi}\left[\sum_{x, y} \frac{1}{2}\left[e^{-\delta \mu} V_{x}^{+}-e^{\delta \mu} V_{x}^{-}\right]+\sum_{x} \tilde{m}_{q} M_{x}\right] \\
& =Z_{\chi} \sum_{x y} \bar{\chi}_{x} G_{x y}^{-1}\left(\tilde{m}_{q} ; \tilde{\mu}, T\right) \chi_{y},
\end{aligned}
$$




$$
\begin{gathered}
V_{x}^{+}=\bar{\chi}_{x} e^{\mu} U_{0, x} \chi_{x+\hat{0}}, \quad V_{x}^{-}=\bar{\chi}_{x+\hat{0}} e^{-\mu} U_{0, x}^{\dagger} \chi_{x}, \\
M_{x}=\bar{\chi}_{x} \chi_{x} .
\end{gathered}
$$

Finite coupling effects result in the modifications of the wave function renormalization factor $Z_{\chi}=\sqrt{Z_{+} Z_{-}}$, quark mass $\tilde{m}_{q}=m_{q}^{\prime} / Z_{\chi}$, and effective chemical potential $\tilde{\mu}=$ $\mu-\delta \mu=\mu-\log \sqrt{Z_{+} / Z_{-}} \cdot Z_{ \pm}$and $m_{q}^{\prime}$ are defined as

$$
\begin{gathered}
m_{q}^{\prime}=b_{\sigma}^{\prime} \sigma+m_{0}-\beta_{\tau \tau}\left(\bar{\psi}_{\tau \tau}+\psi_{\tau \tau}\right), \\
Z_{+}=1+\beta_{\tau}^{\prime} \bar{\psi}_{\tau}+4 \beta_{\tau \tau} m_{q}^{\prime} \bar{\psi}_{\tau \tau}, \\
Z_{-}=1+\beta_{\tau}^{\prime} \psi_{\tau}+4 \beta_{\tau \tau} m_{q}^{\prime} \psi_{\tau \tau} .
\end{gathered}
$$

$S_{\text {eff }}^{(X)}$ represents the auxiliary field part of the effective action,

$$
\begin{aligned}
\mathcal{F}_{\mathrm{eff}}^{(X)}= & S_{\mathrm{eff}}^{(X)} /\left(N_{\tau} L^{d}\right) \\
= & \frac{1}{2} b_{\sigma}^{\prime} \sigma^{2}+\frac{1}{2} \beta_{\tau}^{\prime} \bar{\psi}_{\tau} \psi_{\tau}+\frac{1}{2} \beta_{s}^{\prime} \bar{\psi}_{s} \psi_{s}+\beta_{\tau \tau} \bar{\psi}_{\tau \tau} \psi_{\tau \tau} \\
& +\beta_{s s} \bar{\psi}_{s s} \psi_{s s}+\frac{1}{2} \beta_{\tau s} \bar{\psi}_{\tau s} \psi_{\tau s} .
\end{aligned}
$$

Auxiliary fields $\left(\sigma,\left\{\psi_{K}, \bar{\psi}_{K} ; K=\tau, s, \tau \tau, \tau s, s s\right\}\right)$ in Eq. (A8) are introduced as mean fields for the mesonic composites. We show the relation between auxiliary fields and the mesonic elements in Table IV. The coefficients of the auxiliary field in Eq. (A8) are defined as

$$
\begin{gathered}
b_{\sigma}^{\prime}=b_{\sigma}+2\left[\beta_{s s} \psi_{s s}+\beta_{\tau s} \bar{\psi}_{\tau s}+\beta_{s}^{\prime}\left(\psi_{s}+\bar{\psi}_{s}\right)\right], \\
\beta_{\tau}^{\prime}=\beta_{\tau}+\beta_{\tau s} \psi_{\tau s}, \quad \beta_{s}^{\prime}=\beta_{s}+2 \beta_{s s} \bar{\psi}_{s s}, \\
b_{\sigma}=\frac{d}{2 N_{c}}, \quad \beta_{\tau}=\frac{d}{N_{c}^{2} g^{2}}\left(1+\frac{1}{2 g^{2}}\right) \\
\beta_{s}=\frac{d(d-1)}{8 N_{c}^{4} g^{2}}\left(1+\frac{1}{2 g^{2}}\right), \quad \beta_{\tau \tau}=\frac{d}{2 N_{c}^{3} g^{4}},
\end{gathered}
$$

TABLE IV. The auxiliary fields in the NNLO effective potential. $W_{\mathbf{x}}^{+}$and $W_{\mathbf{x}}^{-}$are represented as the mesonic composite which connects the quark fields in the next-to-nearest neighboring temporal sites $\left(W_{\mathbf{x}}^{+}=\bar{\chi}_{x} e^{2 \mu} U_{0, x} U_{0, x+\hat{0}} \chi_{x+2 \hat{0}}\right.$ and $W_{\mathbf{x}}^{-}=$ $\left.\bar{\chi}_{x+2 \hat{0}} e^{-2 \mu} U_{0, x+\hat{0}}^{\dagger} U_{0, x}^{\dagger} \chi_{x}\right)$. We omit the indices $x$ ( $\mathbf{x}$ in the case of $\ell, \bar{\ell})$ in the mesonic composites.

\begin{tabular}{lc}
\hline \hline Auxiliary fields & Mean fields \\
\hline$\sigma$ & $-M$ \\
$\left(\bar{\psi}_{\tau \tau}, \psi_{\tau \tau}\right)$ & $\left(W^{+}, W^{-}\right)$ \\
$\left(\bar{\psi}_{s s}, \psi_{s s}\right)$ & $(M M, M M M M)$ \\
$\left(\bar{\psi}_{\tau s}, \psi_{\tau s}\right)$ & $\left(-V^{+} V^{-}, 2 M M\right)$ \\
$\left(\bar{\psi}_{\tau}, \psi_{\tau}\right)$ & $\left(-V^{+}, V^{-}\right)$ \\
$\left(\bar{\psi}_{s}, \psi_{s}\right)$ & $(M M, M M)$ \\
$(\bar{\ell}, \ell)$ & $(\bar{P}, P)$ \\
\hline \hline
\end{tabular}

$$
\beta_{s s}=\frac{d(d-1)(d-2)}{16 N_{c}^{7} g^{4}}, \quad \beta_{\tau s}=\frac{d(d-1)}{2 N_{c}^{5} g^{4}} .
$$

\section{APPENDX B: THE TEMPORAL LINK INTEGRAL IN THE WEISS MEAN-FIELD METHOD}

The part of the temporal link integral in Eq. (10) is given as

$$
\begin{aligned}
Z_{P}= & \int d \mathcal{U}_{0} \exp \left[2 \beta_{p}\left(\bar{P}_{\mathbf{x}} \ell+\bar{\ell} P_{\mathbf{x}}\right)\right] e^{N_{c} E_{q} / T} \\
& \times \operatorname{det}_{c}\left\{\left[1+\mathcal{U}_{0} e^{-\left(E_{q}-\tilde{\mu}\right) / T}\right]\left[1+\mathcal{U}_{0}^{\dagger} e^{-\left(E_{q}+\tilde{\mu}\right) / T}\right]\right\} \\
= & \int\left[d \mathcal{U}_{0}\right] \prod_{a=1}^{N_{c}} \exp \left(\eta e^{-i \theta_{a}}+\bar{\eta} e^{i \theta_{a}}\right) \\
& \times\left[X+Y e^{i \theta_{a}}+\frac{e^{-i \theta_{a}}}{Y}\right] .
\end{aligned}
$$

We define $\quad \eta=2 \beta_{p} \ell / N_{c}, \quad \bar{\eta}=2 \beta_{p} \bar{\ell} / N_{c}, \quad X=$ $2 \cosh \left(N_{\tau} E_{q}\right)$, and $Y=\exp \left(N_{\tau} \tilde{\mu}\right)$.

This type of temporal link integral is written for $N_{c}=3$ as [17]

$$
\begin{aligned}
\int\left[d \mathcal{U}_{0}\right] \prod_{a=1}^{N_{c}} f\left(\theta_{a}\right) \\
=\sum_{n=-\infty}^{\infty} N_{c} ! d_{i, j} M_{n+i-j} \\
=N_{c} ! \sum_{n}\left[M_{n}^{3}+M_{n+1}^{2} M_{n-2}+M_{n-1}^{2} M_{n+2}\right. \\
\left.\quad-M_{n}\left(M_{n+2} M_{n-2}+2 M_{n+1} M_{n-1}\right)\right],
\end{aligned}
$$

where

$$
\begin{gathered}
M_{n}=\int_{-\pi}^{\pi} \frac{d \theta}{2 \pi} f(\theta) \exp [-i n \theta], \\
f(\theta)=e^{i z \sin (\theta-\pi / 2-i \phi)}\left[X+Y e^{i \theta}+\frac{e^{-i \theta}}{Y}\right],
\end{gathered}
$$

where $z=2 i \sqrt{\eta \bar{\eta}}$ and $\phi=\frac{1}{2} \log (\bar{\eta} / \eta)$. By using to the relation $e^{i z \sin \theta}=\sum_{l=-\infty}^{\infty} J_{l}(z) e^{i l \theta}$, where $J_{l}(z)$ is the Bessel function and $l, z$ are an integer and a complex number, respectively, $f(\theta)$ is found to be

$$
f(\theta)=\sum_{l=-\infty}^{\infty} J_{l}(z) e^{i l(\theta-\pi / 2-i \phi)}\left[X+Y e^{i \theta}+\frac{e^{-i \theta}}{Y}\right] .
$$

We substitute Eq. (B5) into Eq. (B3) and carry out the integral over $\theta$. The matrix element $M_{n}$ is calculated to be

$$
M_{n}=e^{n \phi}\left[I_{n}(y) X+I_{n-1}(y) e^{-\phi} Y+I_{n+1}(y) \frac{e^{\phi}}{Y}\right]
$$

Note that $I_{\nu}(y)=e^{-\nu \pi i / 2} J_{\nu}(i y)$ is the modified Bessel function, where $y=2 \sqrt{\eta \bar{\eta}}$. By substituting Eq. (6) into Eq. (B2), the temporal integral is found to be [up to a constant $\left.\left(N_{c} !\right)\right]$ 
TAKASHI Z. NAKANO, KOHTAROH MIURA, AND AKIRA OHNISHI

$$
\begin{aligned}
Z_{P}= & D_{1} X^{3}+D_{2} X+D_{3} \tilde{Y}^{3}+D_{-3} \tilde{Y}^{-3} \\
& +X^{2}\left(D_{4} \tilde{Y}+D_{-4} \tilde{Y}^{-1}\right)+X\left(D_{5} \tilde{Y}^{2}+D_{-5} \tilde{Y}^{-2}\right) \\
& +D_{6} \tilde{Y}+D_{-6} \tilde{Y}^{-1},
\end{aligned}
$$

where $\tilde{Y}=Y e^{-\phi}$. Coefficients $D_{i}(i=1,2, \pm 3, \cdots, \pm 6)$ are defined as

$$
\begin{gathered}
D_{i}(\eta, \bar{\eta}) \equiv \sum_{n=-\infty}^{\infty} e^{N_{c} n \phi} D_{n, i} \\
D_{n, 1}=I_{n}^{3}-I_{n+2} I_{n} I_{n-2}-2 I_{n+1} I_{n} I_{n-1} \\
\quad+I_{n+1}^{2} I_{n-2}+I_{n-1}^{2} I_{n+2}, \\
D_{n, 2}=-2\left(I_{n}^{3}-I_{n+2} I_{n} I_{n-2}\right)+5 I_{n+1} I_{n} I_{n-1} \\
-3\left(I_{n+1}^{2} I_{n-2}+I_{n-1}^{2} I_{n+2}\right)-I_{n+3} I_{n} I_{n-3} \\
+I_{n-1} I_{n-2} I_{n+3}+I_{n+1} I_{n+2} I_{n-3}, \\
\quad D_{n, \pm 3}=D_{n \mp 1,1}, \\
D_{n, \pm 4}=I_{n}^{2} I_{n \mp 1}+I_{n \pm 1}^{2} I_{n \mp 3} \\
-I_{n \mp 1}^{2} I_{n \pm 1}+I_{n \pm 2} I_{n \mp 1} I_{n \mp 2}-I_{n \pm 2} I_{n} I_{n \mp 3} \\
\quad-I_{n \pm 1} I_{n} I_{n \mp 2}, \\
\quad D_{n, \pm 5}=D_{n \mp 1, \mp 4}, \\
D_{n, \pm 6}=-\left(I_{n}^{2} I_{n \mp 1}+I_{n \pm 1}^{2} I_{n \mp 3}\right) \\
+2\left(I_{n \mp 1}^{2} I_{n \pm 1}-I_{n \pm 2} I_{n \mp 1} I_{n \mp 2}+I_{n \pm 2} I_{n} I_{n \mp 3}\right) \\
+I_{n \mp 2}^{2} I_{n \pm 3}-I_{n \pm 3} I_{n \mp 1} I_{n \mp 3} .
\end{gathered}
$$

In the numerical calculation, we have confirmed that the sums in $D_{k}$ converge. $D_{n, i}(i=1,2, \pm 3, \cdots, \pm 6)$ are represented as combinations of the modified Bessel functions. From Eqs. (B11) and (B13), $D_{ \pm 3}$ and $D_{ \pm 5}$ are represented as $D_{ \pm 3}=e^{ \pm N_{c} \phi} D_{1}$ and $D_{ \pm 5}=e^{ \pm N_{c} \phi} D_{\mp 4}$. We find $D_{i^{\prime}}(\eta, \bar{\eta})=D_{i}(\bar{\eta}, \eta)$ from the relation $D_{-n, i^{\prime}}=$ $D_{n, i}\left(i^{\prime}=i\right.$ for $i=1,2$ and $i^{\prime}=-i$ for $\left.i=3, \ldots, 6\right)$. By utilizing these properties, $Z_{P}$ is rewritten as

$$
\begin{aligned}
Z_{P} / D_{1}= & X^{3}+Y^{3}+Y^{-3}+\tilde{D}_{2} X \\
& +X^{2}\left(\tilde{D}_{4} Y+\tilde{D}_{-4} Y^{-1}\right)+X\left(\tilde{D}_{-4} Y^{2}+\tilde{D}_{4} Y^{-2}\right) \\
& +\tilde{D}_{6} Y+\tilde{D}_{-6} Y^{-1}, \\
\tilde{D}_{2} \equiv & D_{2} / D_{1}, \quad \tilde{D}_{ \pm k}=D_{ \pm k} e^{\mp \phi} / D_{1}(k=4,6) .
\end{aligned}
$$

When the quarks are confined $(\ell \rightarrow 0, \bar{\ell} \rightarrow 0)$, the zeroth modified Bessel function remains finite $\left(I_{0} \rightarrow 1\right)$ but others vanish, $I_{n}(y) \rightarrow 0(n>0, n<0)$. In this limit, $Z_{P}$ reproduces the standard SC-LQCD expression without the Polyakov loop effects,

$$
\begin{gathered}
Z_{P} \rightarrow X^{3}-2 X+Y^{3}+Y^{-3} \\
=\frac{\sinh \left(\left(N_{c}+1\right) E_{q} / T\right)}{\sinh \left(E_{q} / T\right)}+2 \cosh \left(N_{c} \tilde{\mu} / T\right), \\
D_{1} \rightarrow 1, \quad D_{2} \rightarrow-2 .
\end{gathered}
$$

In this main part, we redefine $L_{0} \equiv D_{1}, L_{1} \equiv \tilde{D}_{4}, \bar{L}_{1} \equiv$ $\tilde{D}_{-4}, L_{2} \equiv \tilde{D}_{2}, L_{3} \equiv \tilde{D}_{6}, \bar{L}_{3} \equiv \tilde{D}_{-6}$.
[1] For a recent review, see B. Müller and J. L. Nagle, Annu. Rev. Nucl. Part. Sci. 56, 93 (2006).

[2] Y. Aoki, G. Endrodi, Z. Fodor, S. D. Katz, and K. K. Szabo, Nature (London) 443, 675 (2006); Y. Aoki, Z. Fodor, S. D. Katz, and K. K. Szabo, Phys. Lett. B 643, 46 (2006); Y. Aoki, S. Borsanyi, S. Durr, Z. Fodor, S. D. Katz, S. Krieg, and K. K. Szabo, J. High Energy Phys. 06 (2009) 088; S. Borsanyi, Z. Fodor, C. Hoelbling, S. D. Katz, S. Krieg, C. Ratti, and K. K. Szabo (WuppertalBudapest Collaboration), J. High Energy Phys. 09 (2010) 073.

[3] For a recent review, see M. G. Alford, A. Schmitt, K. Rajagopal, and T. Schafer, Rev. Mod. Phys. 80, 1455 (2008).

[4] L. McLerran and R. D. Pisarski, Nucl. Phys. A796, 83 (2007); Y. Hidaka, L. D. McLerran, and R. D. Pisarski, Nucl. Phys. A808, 117 (2008).

[5] For a recent review, see F. Karsch, Lect. Notes Phys. 583, 209 (2002).
[6] M. Cheng et al., Phys. Rev. D 74, 054507 (2006); A. Bazavov et al., Phys. Rev. D 80, 014504 (2009).

[7] For a recent review, see S. Muroya, A. Nakamura, C. Nonaka, and T. Takaishi, Prog. Theor. Phys. 110, 615 (2003); F. Karsch, Prog. Theor. Phys. Suppl. 153, 106 (2004).

[8] F. Karsch and K. H. Mutter, Nucl. Phys. B313, 541 (1989); G. Boyd, J. Fingberg, F. Karsch, L. Karkkainen, and B. Petersson, Nucl. Phys. B376, 199 (1992).

[9] G. Münster, Nucl. Phys. B180, 23 (1981).

[10] J. Langelage, G. Munster, and O. Philipsen, J. High Energy Phys. 07 (2008) 036; J. Langelage and O. Philipsen, J. High Energy Phys. 01 (2010) 089; 04 (2010) 055.

[11] J. Polonyi and K. Szlachanyi, Phys. Lett. 110B, 395 (1982); M. Gross, Phys. Lett. 132B, 125 (1983); J. Bartholomew, D. Hochberg, P.H. Damgaard, and M. Gross, Phys. Lett. 133B, 218 (1983). 
[12] P. H. Damgaard, N. Kawamoto, and K. Shigemoto, Phys. Rev. Lett. 53, 2211 (1984); Nucl. Phys. B264, 1 (1986).

[13] G. Faldt and B. Petersson, Nucl. Phys. B265, 197 (1986).

[14] P. H. Damgaard, D. Hochberg, and N. Kawamoto, Phys. Lett. 158B, 239 (1985).

[15] K. Fukushima, Prog. Theor. Phys. Suppl. 153, 204 (2004).

[16] Y. Nishida, K. Fukushima, and T. Hatsuda, Phys. Rep. 398, 281 (2004); N. Kawamoto, K. Miura, A. Ohnishi, and T. Ohnuma, Phys. Rev. D 75, 014502 (2007).

[17] Y. Nishida, Phys. Rev. D 69, 094501 (2004).

[18] V. Azcoiti, G. Di Carlo, A. Galante, and V. Laliena, J. High Energy Phys. 09 (2003) 014.

[19] P. de Forcrand and M. Fromm, Phys. Rev. Lett. 104, 112005 (2010).

[20] K. Miura, T.Z. Nakano, A. Ohnishi, and N. Kawamoto, Phys. Rev. D 80, 074034 (2009); K. Miura, T. Z Nakano, and A. Ohnishi, Prog. Theor. Phys. 122, 1045 (2009).

[21] T.Z. Nakano, K. Miura, and A. Ohnishi, Prog. Theor. Phys. 123, 825 (2010).

[22] N. Bilic, K. Demeterfi, and B. Petersson, Nucl. Phys. B377, 651 (1992); N. Bilic, F. Karsch, and K. Redlich, Phys. Rev. D 45, 3228 (1992); N. Bilic and J. Cleymans, Phys. Lett. B 355, 266 (1995).

[23] T. Jolicoeur, H. Kluberg-Stern, M. Lev, A. Morel, and B. Petersson, Nucl. Phys. B235, 455 (1984).

[24] A. Gocksch and M. Ogilvie, Phys. Rev. D 31, 877 (1985).

[25] E. M. Ilgenfritz and J. Kripfganz, Z. Phys. C 29, 79 (1985).

[26] K. Fukushima, Phys. Lett. B 553, 38 (2003); Phys. Rev. D 68, 045004 (2003).

[27] K. Miura, T.Z. Nakano, A. Ohnishi, and N. Kawamoto, arXiv:1012.1509.

[28] N. Kawamoto and J. Smit, Nucl. Phys. B192, 100 (1981).

[29] H. Kluberg-Stern, A. Morel, and B. Petersson, Nucl. Phys. B215, 527 (1983).
[30] T. Hatsuda and T. Kunihiro, Phys. Rep. 247, 221 (1994).

[31] K. Fukushima, Phys. Lett. B 591, 277 (2004); Phys. Rev. D 77, 114028 (2008).

[32] C. Ratti, M. A. Thaler, and W. Weise, Phys. Rev. D 73, 014019 (2006).

[33] E. V. Shuryak and J. J. M. Verbaarschot, Nucl. Phys. A560, 306 (1993).

[34] K. G. Wilson, Phys. Rev. D 10, 2445 (1974).

[35] J. B. Kogut, M. Snow, and M. Stone, Nucl. Phys. B200, 211 (1982).

[36] N. Brambilla, J. Ghiglieri, P. Petreczky, and A. Vairo, Phys. Rev. D 82, 074019 (2010).

[37] Y. Araki and T. Hatsuda, Phys. Rev. B 82, 121403 (2010); Y. Araki, arXiv:1010.0847.

[38] L. Susskind, Phys. Rev. D 16, 3031 (1977).

[39] H. S. Sharatchandra, H. J. Thun, and P. Weisz, Nucl. Phys. B192, 205 (1981).

[40] P. Hasenfratz and F. Karsch, Phys. Lett. B 125, 308 (1983).

[41] C. R. Allton et al., Phys. Rev. D 66, 074507 (2002); A. Dumitru, R. D. Pisarski, and D. Zschiesche, Phys. Rev. D 72, 065008 (2005); K. Fukushima and Y. Hidaka, Phys. Rev. D 75, 036002 (2007).

[42] B. J. Schaefer, M. Wagner, and J. Wambach, Phys. Rev. D 81, 074013 (2010).

[43] E. Megias, E. Ruiz Arriola, and L. L. Salcedo, Phys. Rev. D 74, 065005 (2006).

[44] P. de Forcrand (private communication).

[45] S. A. Gottlieb, W. Liu, D. Toussaint, R. L. Renken, and R. L. Sugar, Phys. Rev. D 35, 3972 (1987).

[46] M. D'Elia and M. P. Lombardo, Phys. Rev. D 67, 014505 (2003).

[47] Z. Fodor and S. D. Katz, Phys. Lett. B 534, 87 (2002).

[48] R. V. Gavai et al., Phys. Lett. B 241, 567 (1990). 